\title{
Réhabilitation auditive
}

Didier Bouccara, Paul Avan, Isabelle Mosnier, Alexis Bozorg Grayeli, Évelyne Ferrary,

> Les modalités de la réhabilitation auditive Olivier Sterkers dépendent de la cause et de la sévérité de la surdité. Les surdités rattachées à des atteintes de l'oreille moyenne peuvent bénéficier de traitements médicaux ou chirurgicaux, par des prothèses ossiculaires, s'il est nécessaire de restaurer la fonction de la chaîne des osselets. Dans les surdités neurosensorielles, surdités d'oreille interne, l'utilisation d'aides auditives est d'emblée envisagée. Dans les cas pour lesquels elles sont insuffisantes, du fait de la sévérité de la surdité, ou non utilisables, pour des raisons d'intolérance, il est possible de recourir à des prothèses implantées ou à des implants cochléaires. Les indications des implants du tronc cérébral restent à ce jour limitées aux surdités totales bilatérales liées à une destruction complète des cochlées et des nerfs cochléaires. Ces orientations thérapeutiques sont choisies après une évaluation multidisciplinaire du malentendant, qui permet de caractériser l'atteinte auditive et son retentissement. Dans tous les cas, la restauration d'une audition bilatérale est à privilégier, dans le but d'améliorer la compréhension globale, en particulier dans les situations bruyantes, et de localiser les sources sonores. <

\section{Les différents types de surdité}

Les grandes causes de surdité se répartissent en trois cadres bien distincts qui correspondent chacun à une étape physiologique du traitement de l'information sonore, à une classe d'atteintes auditives particulières et à une conduite à tenir radicalement différente. Le $1^{\text {er }}$ cadre concerne l'oreille moyenne et la chaîne tympano-ossiculaire, dont le rôle est de transmettre les vibrations du milieu extérieur (l'air) aux liquides de l'oreille interne. La géométrie particulière de la chaîne apporte un gain de pression d'environ 30 . En son absence, la fraction de puissance acoustique communiquée à

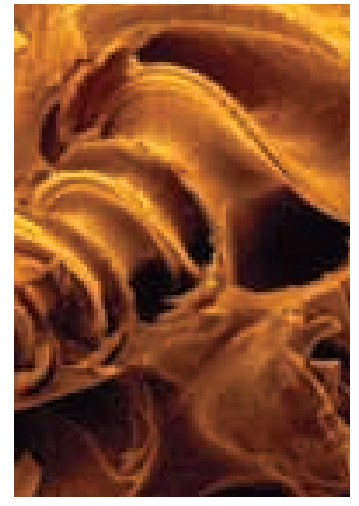

l'oreille interne serait de 1/1 000, mais le gain en pression la fait remonter à une valeur proche de l'unitél. Dans le cas d'une transmission défectueuse, la puissance sonore reçue par l'oreille interne peut chuter d'un facteur 1000 , environ, ce qui entraîne une surdité de transmission légère à moyenne (de 30 décibels typiquement); la sensibilité est diminuée, mais les autres performances auditives ne sont pas affectées. Le $2^{\mathrm{e}}$ cadre est celui des surdités de perception dues à l'oreille interne. Il est le plus fréquemment rencontré lorsqu'un appareillage est envisagé et son examen est développé ci-après. Le $3^{e}$ cadre inclut les surdités dites rétrocochléaires, ou centrales, dues à une atteinte des voies nerveuses entre l'organe sensoriel et le cortex (par exemple, en cas de tumeur). II pose des problèmes de diagnostic précoce, éventuellement de survie en cas d'intervention tardive, ou encore de réhabilitation auditive en cas d'atteinte bilatérale dans le cadre de la neurofi-

\footnotetext{
${ }^{1}$ La gamme d'intensité sonore que l'oreille humaine peut traiter correspond à un rapport de puissance de 10 millions pour 1. Pour cette raison, il est plus commode de décrire l'intensité du son en utilisant une échelle logarithmique et en exprimant un son sous la forme d'un rapport de deux intensités, l'une étant choisie comme la référence. L'unité de mesure utilisée est le Bel, qui correspond à un rapport d'intensité de $10: 1$. De plus, il est apparu souhaitable de comparer la sensibilité auditive d'un individu donné à des valeurs «normales ». Dans ce but, le seuil d'audition est exprimé par rapport à une valeur de référence déterminée à partir d'un groupe de sujets jeunes ayant une audition normale. Lorsque l'audition est testée avec un audiomètre en clinique, le seuil mesuré est exprimé en $\mathrm{dB}$ HL (decibels hearing level).
} 
bromatose de type 2 . En raison de l'atteinte des voies auditives qui affecte le codage des sons, l'intelligibilité de la parole peut disparaître, même si les seuils auditifs semblent peu élevés.

Les surdités de perception, ou surdités neurosensorielles, qui entrent dans le $2^{\mathrm{e}}$ cadre, sont les plus fréquemment rencontrées: par exemple, la presbyacousie, ou surdité due à l'âge, concerne 4 millions de Français. Elles sont principalement d'origine cochléaire, même si des facteurs centraux peuvent coexister, notamment dans la presbyacousie. L'atteinte des cellules sensorielles cochléaires est irréversible, ces cellules ne pouvant se régénérer lorsqu'elles sont détruites. L'étude des surdités neurosensorielles nécessite de connaître le fonctionnement normal et pathologique de la cochlée, organe de l'audition.

\section{Physiologie de l'audition}

La cochlée abrite l'organe sensoriel de l'audition, l'organe de Corti, qui repose sur la membrane basilaire, structure mise en mouvement par les vibrations sonores transmises par l'oreille moyenne. Les cellules sensorielles analysent le son et le codent sous forme de potentiels d'action, véhiculés ensuite par les neurones auditifs en direction des centres. Deux types de cellules sensorielles sont individualisés: les cellules ciliées internes $(\mathrm{CCl})$, alignées en une rangée, et les cellules ciliées externes $(C C \varepsilon)$, réparties en trois rangées le long de la membrane basilaire. Les $\mathrm{CCl}$ sont les véritables cellules sensorielles auditives: elles relaient les messages vers les neurones afférents, chaque neurone codant une fréquence particulière. Le rôle des CCE est plus complexe: bien qu'elles soient très peu reliées aux neurones afférents, leur destruction (par le bruit, un médicament ototoxique, le vieillissement...) entraîne une surdité.

Les CCE ont un rôle moteur : elles réagissent mécaniquement à la cadence du son excitateur, modulant ainsi la vibration sonore. L'action des CCE a été anticipée dès 1948 par T. Gold: elles participent à une boucle, dite régénérative, qui contribue à amplifier le stimulus en compensant les pertes énergétiques dues aux
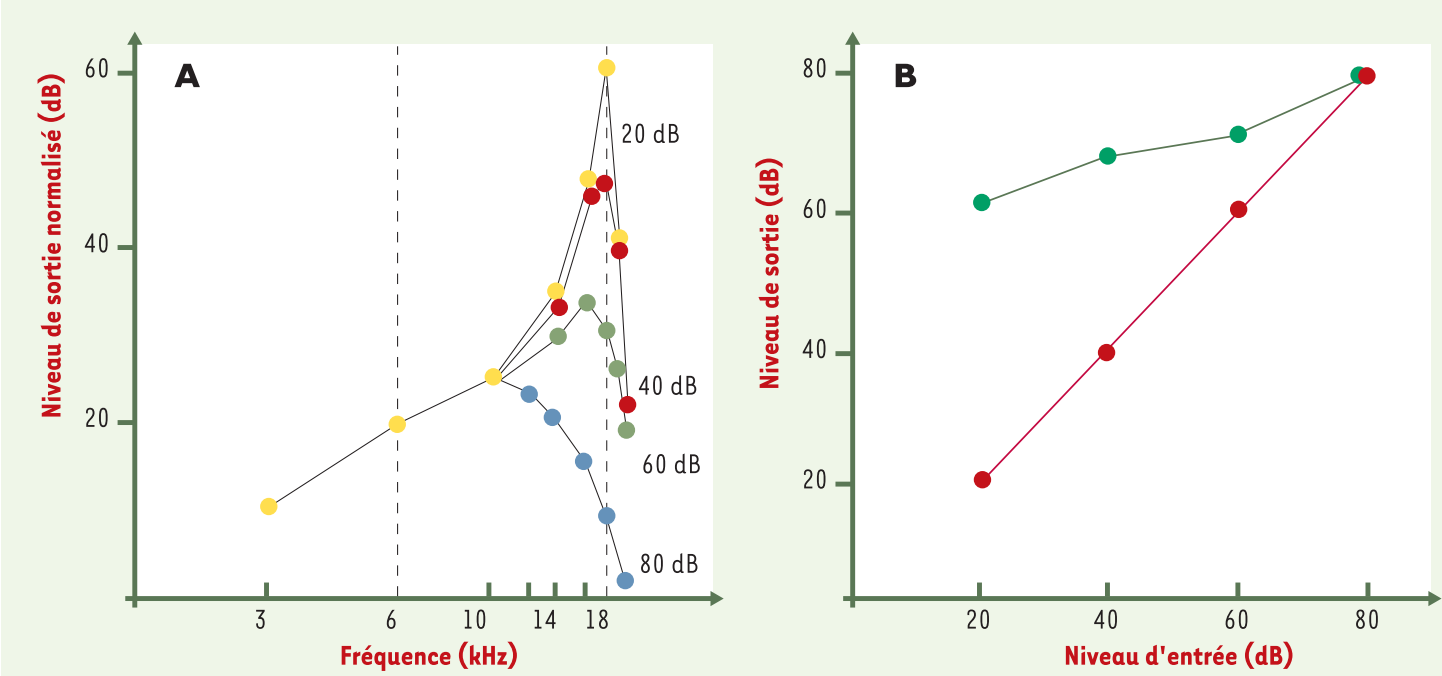

Figure 1. A. Courbes d'accord normale et pathologique. Déplacement de la membrane basilaire d'un animal en parfait état physiologique, mesuré en un point de la membrane dont la meilleure fréquence est $18 \mathrm{kHz}$. Lorsque la stimulation est faible (20 dB SPL [decibels sound pressure level], courbe du haut), la résonance de la membrane est très ample et très fine autour de $18 \mathrm{kHz}$, témoignant de la grande sensibilité de la cochlée, ainsi que de sa sélectivité en fréquence. Au fur et à mesure que la stimulation augmente, par tranche de $20 \mathrm{~dB}$ (jusqu'à $80 \mathrm{~dB}$ SPL), les courbes brutes s'empilent les unes sur les autres, mais la normalisation appliquée retire $20 \mathrm{~dB}$ aux réponses chaque fois que le niveau d'entrée augmente de $20 \mathrm{~dB}$. Cette normalisation est efficace en dessous de $10 \mathrm{kHz}$ puisque, comme cela est souhaité, toutes les courbes normalisées coïncident. Mais c'est autour de $18 \mathrm{kHz}$ que le phénomène intéressant se produit: la résonance devient moins ample et moins fine lorsque le niveau augmente, et les courbes normalisées se décalent vers le bas. La courbe à $80 \mathrm{~dB}$ n'a plus qu'une résonance large, qui, du reste, culmine à $10 \mathrm{kHz}$ et non plus $18 \mathrm{kHz}$. II faut noter qu'après destruction des CCE (cellules ciliées externes) de l'animal, toutes les courbes de 20 à $80 \mathrm{~dB}$ SPL se superposent alors à celle de $80 \mathrm{~dB}$ enregistrée chez l'animal intact (adapté d'après [19]). B. Courbes de croissance normale et pathologique. Les mêmes données qu'en $A$ sont représentées, mais en fonction du niveau d'entrée, à fréquence fixe (soit $6 \mathrm{kHz}$, courbe rouge, soit $18 \mathrm{kHz}$, courbe verte - voir aussi $A$ ). La courbe à $6 \mathrm{kHz}$ est linéaire et de pente $1 \mathrm{~dB} / 1 \mathrm{~dB}$, mais celle à $18 \mathrm{kHz}$ présente une forte compression puisqu'elle diminue peu lorsque le niveau d'entrée diminue: de moins de $20 \mathrm{~dB}$, lorsque l'entrée passe de 80 à $20 \mathrm{~dB}$, cela grâce au gain apporté par les CCE. Les neurones auditifs peuvent ainsi coder les sons de $18 \mathrm{kHz}$ sans saturation dans une large gamme de niveaux. Chez l'animal dont les CCE ont été lésées, et qui a perdu son gain (et sa résonance fine), les courbes sont aussi linéaires à $18 \mathrm{kHz}$ qu'à $6 \mathrm{kHz}$, et les neurones codant pour $18 \mathrm{kHz}$ voient leur dynamique efficace très réduite: la sensation véhiculée paraît souvent avoir une force excessive. Gain, résonance et compression sont trois performances qui vont donc de pair avec des CCE efficaces. 
frottements qui émoussent la résonance de la membrane basilaire [1]. Cette amplification atteint un facteur 100 à 1000 , mais uniquement à un endroit donné de la membrane basilaire, c'est-à-dire à une fréquence donnée (la base de la cochlée est dévolue aux fréquences aiguës, l'apex aux fréquences graves) (Figure 1A). En conséquence, la sensibilité s'accompagne automatiquement d'une discrimination et d'une sélectivité en fréquence excellentes. Inversement, quand les CCE sont lésées, la sensibilité et la sélectivité en fréquence disparaissent de concert, ainsi que la capacité de séparer le signal du bruit ambiant : la surdité (d'environ 40 à 60 décibels) est compensable par un appareillage externe, mais pas la baisse d'intelligibilité (liée à la baisse de sensibilité et de sélectivité en fréquence), qui posera des problèmes de réglage de l'aide auditive. Ces troubles de la compréhension peuvent être prévus par l'évaluation objective de l'activité motrice des CCE : en réponse aux stimulus sonores, les CCE émettent des sons qui peuvent être aisément recueillis dans le conduit auditif externe sous la forme d'oto-émissions acoustiques. II s'agit d'un test de routine qui permet, en outre, un dépistage néonatal de surdité.

Les CCE jouent un rôle supplémentaire, essentiel, de compression au bénéfice des neurones auditifs. Ceux-ci ont intrinsèquement une dynamique faible, passant rapidement du seuil de réponse à la saturation, alors qu'ils doivent coder des sons extérieurs d'intensité très variable. Néanmoins, du fait de l'activité des CCE, la courbe qui relie l'intensité sonore à l'amplitude de la réponse est aplatie (compressive), le neurone codant une gamme d'intensité large (Figure 1B). II en résulte que - en cas de lésion des CCE - les réponses des neurones augmentent trop vite, entraînant une distorsion de la sensation d'intensité sonore: le sujet se plaint davantage des sons forts produits par l'entourage que de ne pas entendre les sons faibles (signe précurseur d'une surdité cochléaire).

Considérant les deux types de cellules sensorielles - CCl et CCE une surdité de perception d'origine cochléaire peut être d'origine « motrice » en cas de lésion des CCE, « sensorielle » si les CCI sont abîmées ou, le plus souvent, mixte, «motrice et sensorielle ». À la surdité s'associent typiquement des troubles de l'intelligibilité, des difficultés de perception dans le bruit et des distorsions. Les conséquences perceptives d'un dysfonctionnement cochléaire dépendent de la (des) structure(s) atteinte(s); il en est de même des réglages d'un appareillage auditif.

On dispose maintenant d'une compréhension de la physiologie de l'organe auditif périphérique et de tests fonctionnels quasi non invasifs qui devraient permettre d'affiner la mise en œuvre d'une réhabilitation auditive efficace. Néanmoins, cette compréhension ne doit pas faire oublier la présence d'un protagoniste, le cerveau, qui, même âgé, possède des capacités étonnantes d'adaptation à condition de ne pas les laisser inutilisées trop longtemps: l'appareillage précoce de cas difficiles peut aboutir à de bons résultats fonctionnels, tandis qu'un appareillage trop tardif ou chez un patient peu motivé peut échouer malgré des réglages techniquement adéquats. 0 r, l'évaluation prédictive des capacités plastiques cérébrales reste à inventer.

\section{Prothèses ossiculaires: dans les cas d'atteinte de l'oreille moyenne}

Les surdités rattachées à l'oreille moyenne sont principalement d'origine inflammatoire (otite chronique avec ou sans lyse ossiculaire), dégénérative (otospongiose), malformative ou traumatique (luxation associée ou non à une fracture ossiculaire après un traumatisme du rocher). Les phénomènes inflammatoires bénéficient le plus souvent d'un traitement médicamenteux, mais doivent, dans certains cas, être éradiqués au cours d'une intervention chirurgicale. En cas d'altération de la chaîne des osselets, la continuité de cette chaîne est rétablie soit en utilisant les restes ossiculaires (ossiculoplastie), soit en utilisant une prothèse qui peut remplacer un ou plusieurs osselets (différents matériaux et formes sont disponibles). Les qualités requises pour ces prothèses ossiculaires sont la bonne stabilité du montage, son efficacité en termes de gain auditif et sa tolérance à long terme, avec l'absence d'extrusion. L'otospongiose est un cas particulier: dans cette affection, il existe une ankylose de l'étrier, qui peut être remplacé par un piston millimétrique en téflon [2].

\section{Aides auditives « conventionnelles 》: dans les cas d'atteinte de l'oreille interne}

Dans le cadre des surdités neurosensorielles, une aide auditive $^{2}$ (audioprothèse) (Figure 2) est proposée après une évaluation multidisciplinaire du malentendant, qui associe, au médecin traitant et à l'oto-rhino-laryngologiste, un audioprothésiste, un orthophoniste et un psychologue. Cette évaluation permet de caractériser l'atteinte auditive et son retentissement, ainsi que les moyens de communication associés qui ont été développés par le sujet malentendant, en particulier l'utilisation de la lecture labiale.

\section{Technologies analogique et numérique}

Le principe de fonctionnement analogique reposait sur la transformation des modifications de la membrane d'un microphone liées à l'énergie sonore en courant électrique. Ce courant était modifié par différents composants (résistances, transistors...) et transformé en énergie acoustique restituée par un écouteur. Les progrès tech-

\footnotetext{
${ }^{2}$ Le terme prothèse auditive, habituellement utilisé, devrait être réservé aux implants cochléaires et du tronc cérébral et aux pistons et appareillages utilisés pour le remplacement des osselets dans la chirurgie de l'oreille moyenne. Tous les autres appareillages qui sont destinés à suppléer ou à corriger la fonction auditive déficiente devraient être appelés orthèses, terme peu utilisé en correction auditive. Les termes « appareils de correction auditive » ou, plus concis, « appareil auditif » et « aide auditive » sont corrects.
} 
nologiques ont abouti au développement de modèles disposant d'un traitement numérique des signaux sonores, privilégiant la parole humaine. Les aides auditives numériques comportent un microphone, un convertisseur analogique digital qui transforme le signal sur un mode binaire, alors traité par un microprocesseur avant d'être transformé par un convertisseur digital analogique. Les différents algorithmes du microprocesseur vont permettre l'accès à une multitude de réglages possibles, adaptés à tous les types de surdité. Des étapes d'évaluation et de suivi régulier sont indispensables pour que le malentendant puisse bénéficier des apports technologiques liés à l'audioprothèse numérique [3]. Les principaux avantages apportés par cette technologie numérique sont:

- la multiprogrammation: le patient peut disposer de différents réglages, adaptés bien sûr à son atteinte auditive mais également aux milieux sonores qu'il est amené à fréquenter;

- l'identification de la voix: la comparaison des timbres captés avec ceux mémorisés permet d'adapter l'amplification de chaque canal pour privilégier les signaux liés à la parole par rapport à ceux liés au bruit. De plus, les réglages permettent de réduire l'amplification si aucun signal vocal n'est capté: en l'absence de parole, le patient sera moins gêné par les bruits environnants;

- la compression multicanaux: en présence de bruits de forte intensité, l'amplification de la zone fréquentielle en cause est sélectivement réduite, sans modification de celle des canaux où l'intensité est faible, d'où un meilleur confort;

- I'évolutivité des réglages: au fur et à mesure de l'utilisation de l'aide auditive, les réglages sont adaptés avec une habituation progressive à des amplifications croissantes;

- la réduction de l'effet Larsen, en particulier par la réduction de l'amplification dans le canal où cet effet est localisé.

Ces possibilités d'amélioration du gain obtenu par les aides numériques ont leurs limites: celles de l'oreille interne du patient, notamment en rai- son des phénomènes de distorsion. II importe donc d'informer le patient sur l'absence de récupération d'une audition normale et sur la nécessité d'un suivi régulier permettant de compenser au mieux l'aggravation progressive de la surdité.

\section{Modalités de prescription et de suivi}

La difficulté essentielle de la prescription d'une aide auditive est d'en définir le bénéfice réel pour le patient malentendant [4]: I'aide auditive ne doit pas finir au fond du tiroir de la table de nuit. Les éléments à prendre en compte sont l'intensité du déficit (seuils mesurés en audiométrie tonale), le seuil d'intelligibilité (audiométrie vocale), les éventuelles atteintes centrales, en particulier l'altération des fonctions d'attention et de mémorisation, mais également la demande du patient qui peut être précise (amélioration de la compréhension en situation bruyante, en langue étrangère...) ou peu personnalisée (les voisins se plaignent du niveau sonore de la télévision). Des tests complémentaires et des questionnaires d'auto-évaluation sont alors utiles pour préciser cette demande [5]. L'audioprothésiste les utilisera pour sa première adaptation des aides auditives. L'utilisation d'un appareillage bilatéral est la règle, avec un bénéfice démontré à moyen et long termes. L'《 accompagnement »du patient durant les premières semaines d'adaptation permet de le guider, lui et son entourage, face aux difficultés possibles. Le réglage optimal est en effet rarement immédiat. Le suivi de l'évolution de la surdité et du bénéfice audioprothétique permet de déceler les limites de ce dernier.
$\mathbf{A}$

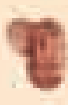

CIC

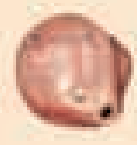

Canal

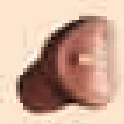

Mini-canal

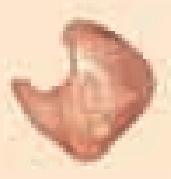

Conque
$\mathbf{B}$

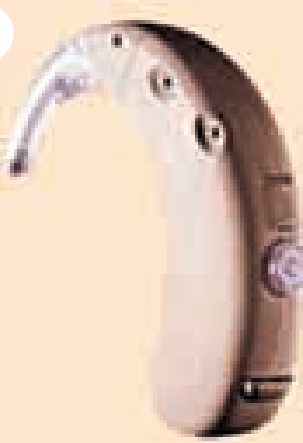

Figure 2. Modèles d'aide auditive conventionnelle. A. Les appareils intra-auriculaires sont constitués d'une coque réalisée sur mesure qui contient tous les composants électroniques. Ils peuvent être placés dans la conque, à l'entrée du conduit auditif externe (canal ou mini-canal) ou dans le conduit auditif externe (intracanal profond, CIC: completely in the canal). B. Les éléments constitutifs des contours d'oreille sont rassemblés dans le boîtier porté derrière le pavillon de l'oreille. L'écouteur est couplé à l'oreille externe par un coude, un tube acoustique et un embout auriculaire réalisé à partir d'une empreinte de l'oreille (d'après [20], avec l'autorisation du CCA).
L'échec d'un appareillage audioprothétique peut résulter de différentes causes. Il peut tout d'abord s'agir d'altérations importantes de l'oreille interne définies comme des zones cochléaires mortes. Ce concept, établi par B. Moore [6], met en évidence la disparition des cellules ciliées sur une zone plus ou moins étendue de l'oreille interne. Celleci peut s'avérer difficile à objectiver en audiométrie car, schématiquement, les zones cochléaires restantes peuvent répondre à une stimulation destinée aux zones mortes. Une chute importante de la courbe audiométrique sur les fréquences aiguës peut faire suspecter l'existence d'une zone morte et faire réaliser des tests spécifiques qui, du fait de leur longueur, n'ont pas été réalisés 
en première intention. La deuxième cause d'échec est la présence d'altérations des voies auditives centrales, associées ou non à des maladies neurologiques [7-9]. Un certain nombre de tests permettent de les dépister et de les identifier. Enfin, l'échec de l'appareillage peut résulter de la sévérité ou de l'évolutivité de la surdité. Dans ces conditions d'échec, d'autres techniques de réhabilitation auditive seront envisagées.

\section{Implants d'oreille moyenne: dans les cas de contre-indication ou d'insuffisance d'amplification de l'aide auditive conventionnelle}

Le but de ces implants est de transmettre à la cochlée toujours fonctionnelle un signal mécanique avec une amplification supérieure à celle d'une aide auditive conventionnelle, et un traitement du signal plus fin. Ils comportent un microphone, un processeur et un transducteur, le plus souvent électromagnétique, qui convertit le signal électrique en vibrations mécaniques; le transducteur est placé chirurgicalement au niveau de la chaîne des osselets (Figure 3) $[10,11]$. Ces implants d'oreille moyenne sont indiqués dans les échecs des aides auditives conventionnelles malgré un réglage optimal, mais également dans leurs contre-indications, en particulier si le port d'un embout dans le conduit auditif externe est impossible (eczéma, otite externe, sténose...). Leur mise en place nécessite une intervention chirurgicale dite fonctionnelle, par un otologiste spécialisé, garantissant le respect de la chaîne des osselets avec une préservation des seuils auditifs et l'absence de complication locale. II s'agit d'une technique relativement récente et un suivi à long terme des patients ayant un implant d'oreille moyenne est en cours, ayant pour objectif de confirmer la bonne tolérance de l'implant et la permanence du bénéfice obtenu.

\section{Implants cochléaires: dans les surdités sévères et profondes}

Les implants cochléaires (Figure 4A) sont indiqués dans les formes les plus sévères des atteintes auditives (surdités bilatérales profondes, supérieures à $90 \mathrm{~dB}$ ) pour lesquelles le bénéfice obtenu avec les aides auditives les plus puissantes est faible ou nul. Compte tenu de son prix (Encadré), l'implant cochléaire est habituellement posé d'un seul côté, le choix se portant le plus souvent sur l'oreille pour laquelle la surdité est la plus récente ou la plus rapidement évolutive. Un porte-électrodes est placé chirurgicalement dans la cochlée. II reçoit par voie transcutanée les stimulations électriques qui correspondent au traitement du signal sonore par un processeur externe. Ces stimulations activeront les voies auditives (qui persistent dans la cochlée un certain temps après la destruction des cellules sensorielles) et permettront une reconnaissance du message vocal par le patient [12]. Une rééducation orthophonique, utilisant la lecture labiale, permet au patient de décoder les nouveaux messages perçus et d'identifier les mots. Les nouveaux processeurs des implants cochléaires s'accompagnent d'un bénéfice rapide chez les adultes devenus sourds.

Chez les enfants présentant une surdité congénitale, l'implantation cochléaire est indiquée précocement, dans les premières années de vie, et, pour certaines équipes, dès la première année, afin de limiter au maximum la durée de privation auditive centrale et de permettre le

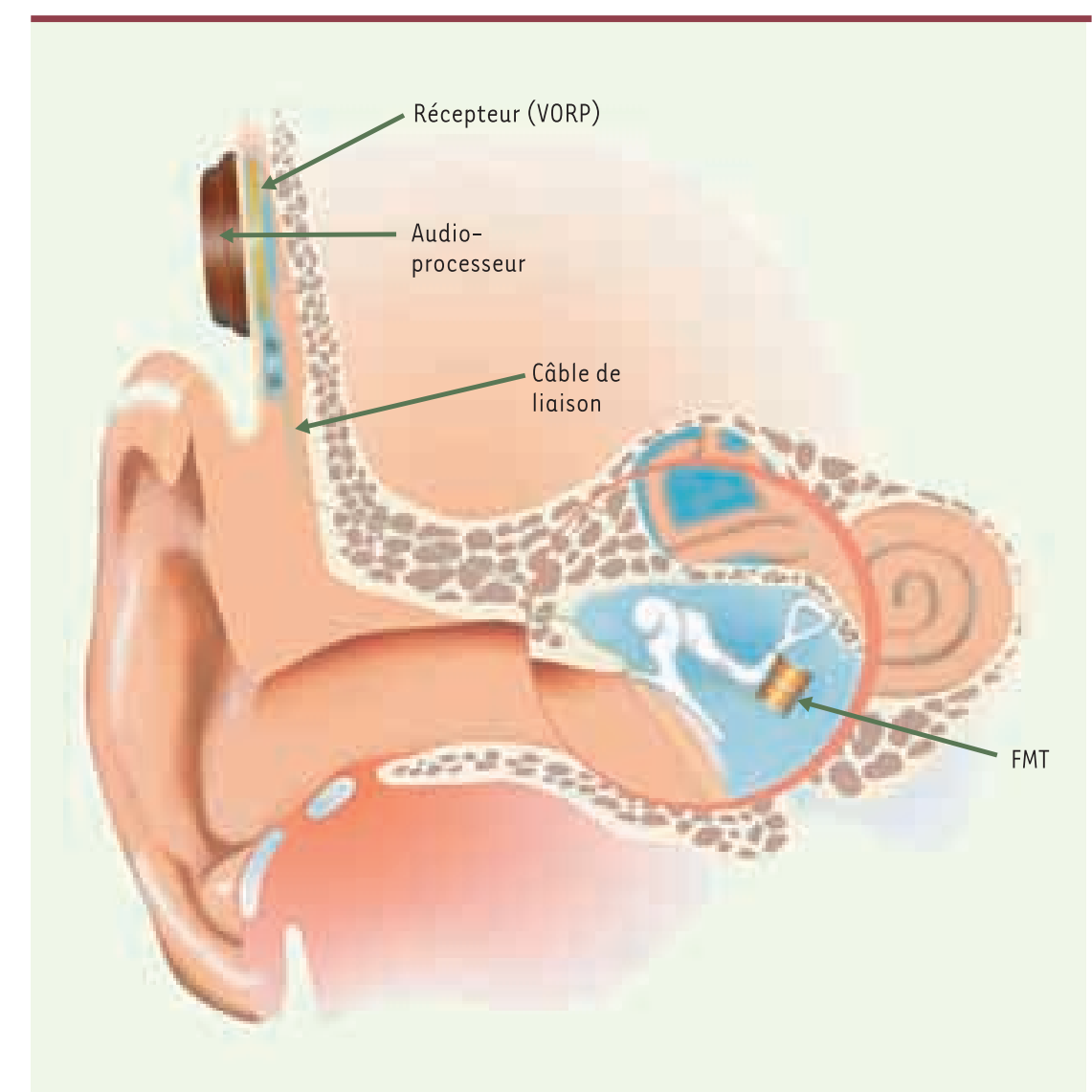

Figure 3. Implants d'oreille moyenne. Ils sont implantés dans l'oreille moyenne, couplés à l'enclume à laquelle ils transmettent les vibrations amplifiées par un système électromagnétique ou piézoélectrique. Ils comportent une partie non encore implantable à ce jour, comprenant le microphone, le processeur vocal et la source d'énergie. FMT: floating mass transducer; VORP : vibrating ossicular prosthesis (d'après [20], avec l'autorisation de Vibrant Medel). 


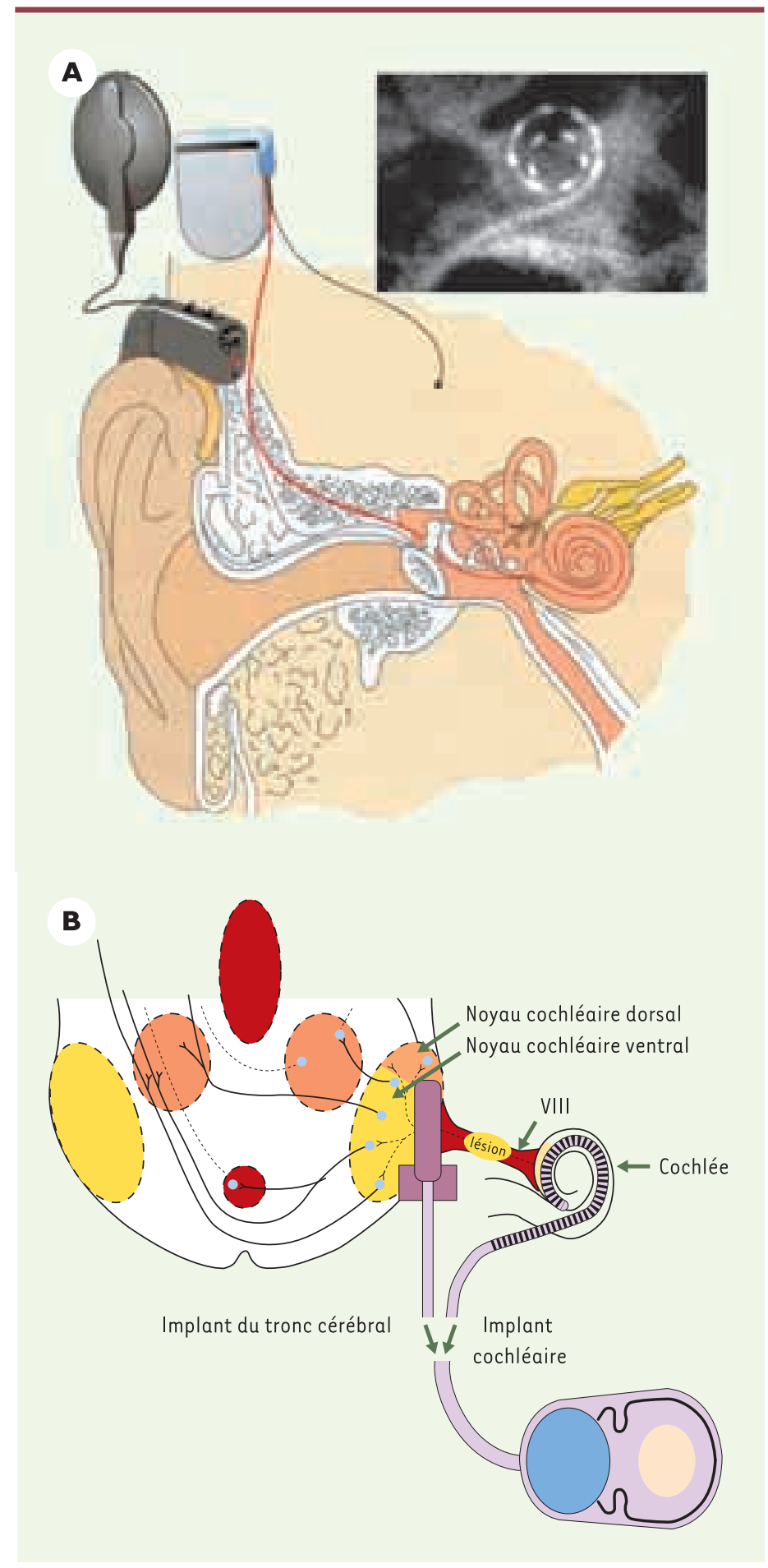

Figure 4. Implants cochléaire et du tronc cérébral. Ces implants se composent d'une partie externe (microphone et microprocesseur réunis dans un contour d'oreille) et d'une partie implantée soit dans la cochlée, soit dans le noyau cochléaire du tronc cérébral. A. Implant cochléaire (avec l'autorisation de Collin Medel). B. Positions respectives de l'implant cochléaire et de l'implant du tronc cérébral (avec l'autorisation de Cochlear). développement du langage. Le diagnostic de surdité profonde ou totale doit donc être rapidement et clairement établi, en particulier avec le support d'examens électrophysiologiques (recueil des potentiels évoqués auditifs, enregistrement des oto-émissions...). Contrairement à l'adulte devenu sourd chez qui le bénéfice dû à l'implant cochléaire est acquis en quelques semaines, chez l'enfant présentant une surdité prélinguale (avant l'acquisition du langage), le développement du langage implique une prise en charge multidisciplinaire avec une rééducation orthophonique intensive.

Les bons résultats obtenus avec les implants cochléaires ont contribué à en élargir les indications [13], en particulier dans le but de restaurer une audition bilatérale. Chez un adulte présentant une surdité évolutive, il paraît actuellement licite de proposer une implantation cochléaire dès que, pour l'un des côtés, l'aide auditive la plus puissante ne lui apporte aucun bénéfice. L'implantation de ce côté permettra, avec l'aide auditive controlatérale, une stimulation auditive bimodale dont le bénéfice est supérieur à celui obtenu avec un seul des deux procédés [14]. Par ailleurs, en cas d'absence de bénéfice d'aucune des deux aides auditives chez un sourd profond, une implantation cochléaire bilatérale peut être proposée. Elle permet d'améliorer les performances en termes de compréhension dans le calme et en atmosphère bruyante, et de mieux localiser les sources sonores [15]. Une des limites avancées est le coût des implants cochléaires (Encadré), mais les études évaluant le rapport coût/bénéfice indiquent que l'implantation cochléaire bilatérale est justifiée sous réserve d'une indication correctement posée [16]. Une autre des nouvelles indications de l'implant cochléaire bilatéral est la surdité post-méningitique, au cours de laquelle une ossification cochléaire peut survenir et limiterait les possibilités ultérieures d'implantation.

Plus récemment, se sont développés des implants dits « hybrides», ou «électro-acoustiques», qui combinent une double stimulation, électrique pour les fréquences aiguës (comme un implant cochléaire) et acoustique pour les fréquences graves (comme une aide auditive). Ils sont indiqués dans les surdités importantes portant sur les fréquences aiguës, en particulier avec des zones cochléaires mortes, tandis que des restes auditifs persistent sur les fréquences graves. Leur utilisation est possible grâce à une adaptation de la technique chirurgicale avec un modèle de porteélectrodes non traumatisant pour les structures résiduelles de l'oreille interne. 


\section{Implants du tronc cérébral : en cas d'atteinte bilatérale du nerf auditif}

Certaines maladies rendent impossible l'implantation cochléaire. C'est le cas de la neurofibromatose de type 2, au cours de laquelle le développement d'un schwannome vestibulaire bilatéral peut entraîner une surdité totale bilatérale. L'obstacle anatomique étant en aval de la cochlée, sur les voies auditives, l'implant cochléaire est inefficace. II est possible de proposer une implantation des noyaux cochléaires du tronc cérébral. Le principe de fonctionnement de l'implant du tronc cérébral est le même que celui de l'implant cochléaire, la seule différence concerne le porte-électrode qui est adapté à l'anatomie du tronc (Figure 4B). L'implant est généralement placé lors de l'intervention neurochirurgicale d'exérèse de l'un des schwannomes vestibulaires. Il s'agit d'une solution thérapeutique qui permet de proposer une réhabilitation auditive à des patients jeunes, sourds profonds - ne pouvant pas bénéficier des autres techniques d'aide auditive - et souffrant fréquemment d'autres atteintes neurologiques et sensorielles rattachées à l'extension de la maladie.

\section{Prothèses à ancrage osseux: dans des indications très particulières}

Les os de la voûte crânienne ont la propriété de transmettre les vibrations osseuses jusqu'à l'oreille interne. Cette propriété est utilisée par certaines prothèses comprenant un pilier en titane placé chirurgicalement dans l'os crânien (Figure 5). Ce pilier va s'intégrer dans I'os en plusieurs semaines. Une prothèse auditive de type « conventionnelle » est alors adaptée au pilier: les vibrations amplifiées sont directement transmises par voie osseuse aux deux oreilles internes. Ces prothèses ont deux indications distinctes: les atteintes de l'oreille moyenne, pour lesquelles il n'est pas possible de restaurer la fonction des osselets, l'oreille interne étant normale, mais la prothèse auditive conventionnelle n'apportant pas un bénéfice suffisant ou étant mal tolérée, et les surdités totales unilatérales, pour lesquelles les informations sonores venant du côté atteint seront transmises par conduction osseuse au côté sain, permettant ainsi de récupérer, au moins partiellement, des informations auditives bilatérales [17, 18].

\section{Conclusions}

Les techniques actuellement disponibles, de l'audioprothèse conventionnelle à l'implant auditif du tronc cérébral, permettent de proposer une réhabilitation auditive quelles que soient la cause et la sévérité de l'atteinte auditive. Pour être efficace, cette suppléance doit être adaptée à chaque patient. Les résultats dépendent des capacités d'adaptation du sujet et de l'utilisation qu'il fait de son aide auditive; ils peuvent nécessiter de renforcer d'autres modes de communication tels que la lecture labiale. La restauration d'une audition bilatérale doit être privilégiée, le plus précocement possible. La prise en charge multidisciplinaire contribue à améliorer le suivi, l'adaptation et l'évaluation des bénéfices obtenus. $\diamond$

\section{SUMMARY}

\section{Auditory rehabilitation}

Auditory rehabilitation depends of the cause and the severity of the hearing loss (or deafness). Hearing losses dues to middle ear pathologies can beneficiate of medical or surgical treatments, by ossicular prostheses, if it is necessary to restore the function of the ossicles chain. In the sensorineural hearing losses, with inner ear pathology, the use of auditory aid is immediately considered. In the cases for which they are insufficient because of severity of the hearing loss or not suitable because of local non-tolerance, it is possible to use middle ear implant or cochlear implant. The indications of the auditory brainstern implants remain at this day limited to the total bilateral hearing losses due to a complete destruction of cochleae and auditory nerves. These therapeutic orientations are selected after a multidisciplinary evaluation of the deaf person, evaluation that allows the characterization of the hearing loss and its repercussion. In all the cases, the restoration of a bilateral hearing has to be done if possible, making an improvement of the speech comprehension, mainly in the noisy situations, as well as the localization of the sound sources. $\diamond$

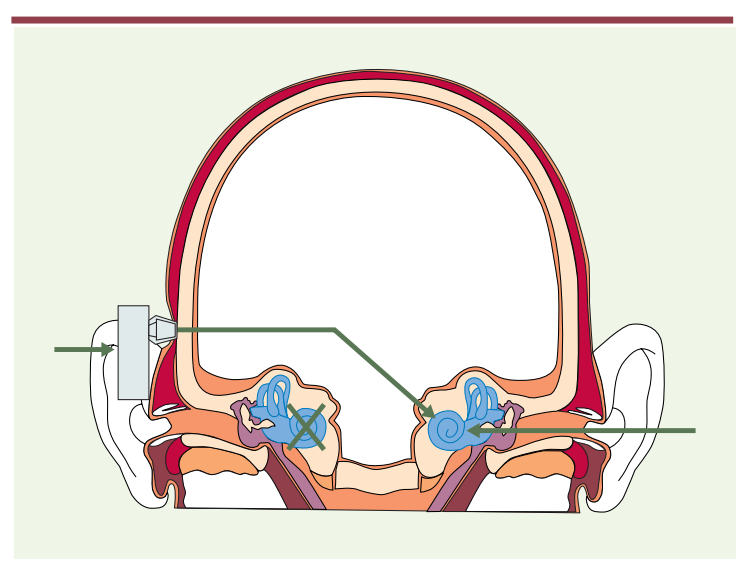

Figure 5. Prothèses à ancrage osseux. II s'agit de prothèses à conduction osseuse directe, stimulant l'os crânien pour la transmission de l'onde sonore vers la cochlée: elles sont homolatérales, pour des patients qui ne peuvent bénéficier d'une aide auditive conventionnelle, ou controlatérales (représenté sur cette figure), si la cochlée homolatérale est détruite ou non fonctionnelle (d'après [20], avec l'autorisation d'Entific). 


\section{RÉFÉRENCES}

1. Gold T. Hearing. The physical basis of the action of the cochlea. Proc $R$ Soc Edinburgh Biol Sci 1948; 135: 492-8.

2. Erminy M, Bonfils P, Trotoux J. Otospongiose. Encycl Med Chir, Elsevier, Paris, Oto-Rhino-Laryngologie 20-195-A-10, 1996, 12 p.

3. Morgon A. L'aide auditive. Encycl Med Chir, Elsevier, Paris, Oto-RhinoLaryngologie 20-185- $-10,1999,11 \mathrm{p}$.

4. Bille M, Parving A. Expectations about hearing aids: demographic and audiological predictors. Int / Audiol 2003; 42: 481-8.

5. Cox RM, Alexander GC. The abbreviated profile of hearing aid benefit. Ear Hear 1995; 16: 176-86.

6. Moore BC, Huss M, Vickers DA, et al. A test for the diagnosis of dead regions in the cochlea. BrJ Audiol 2000; 34: 205-24.

7. Mom T, Avan P, Gilain L. Atteintes centrales de l'audition. Encycl Med Chir, Elsevier, Paris, Oto-Rhino-Laryngologie 20-184-A-10,2001, 10 p.

8. Martini A, Mazzoli M, Rosignoli M, et al. Hearing in the elderly: a population study. Audiology 2001; 40: 285-93.

9. Demanez L, Demanez JP. Central auditory processing assessment. Acta Oto-Rhino-Laryngol Belg 2003; 57: 243-52.

10. Sterkers 0 , Boucarra D, Labassi S, et al. A middle ear implant, the Symphonix Vibrant soundbridge: retrospective study of the first 125 patients implanted in France. Otol Neurotol 2003; 24: 427-36.

11. Kasic JF, Fredrickson JM. The otologics MET ossicular stimulator. Otolaryngol Clin North Am 2001; 34: 501-13.

12. Dauman $\mathrm{R}$, Carbonnière $\mathrm{B}$, Soriano $\mathrm{V}$, et al. Implants cochléaires chez l'adulte et l'enfant. Encycl Med Chir, Elsevier, Paris, Oto-RhinoLaryngologie 20-185-D-10,1998, 12 p.

13. Cohen NL. Cochlear implant candidacy and surgical considerations. Audiol Neurotol 2004; 9 : 197-202.

14. Hamzavi J, Pok SM, Gstoettner W, Baumgartner WD. Speech perception with a cochlear implant used in conjunction with a hearing aid in the opposite ear. Int J Audiol 2004; 43: 61-5.

15. Muller J, Schon F, Helms J. Speech understanding in quiet and noise in bilateral users of the MED-EL COMBI 40/40 + cochlear implant system. Ear Hear 2002; 23: 198-206

16. Summerfield AQ, Marshall DH, Barton GR, Bloor KE. A cost-utility scenario analysis of bilateral cochlear implantation. Arch Otolaryngol Head Neck Surg 2002; 128: 1255-62.

17. Vaneecloo FM, Hanson JN, Laroche C, et al. Prosthetic rehabilitation of unilateral anakusis. Study with stereoaudiometry. Ann Otolaryngol Chir Cervicofac 2000; 117: 410-7.

18. Niparko JK, Cox KM, Lustig LR. Comparison of the bone anchored hearing aid implantable hearing device with contralateral routing of offside signal amplification in the rehabilitation of unilateral deafness. Otol Neurotol 2003; $24: 73-8$

19. Sellick PM, Patuzzi R, Johnstone BM. Measurement of basilar membrane motion in the guinea pig using the Mossbauer technique. J Acoust Soc Am 1982; 72: 131-41.

20. Casenave A, Mondain M, Frachet B, Hamann C, Sterkers O. Les surdités: de la prothèse à l'implant. Les monographies du CCA. Paris: Éditions Amplifon, 2002.

\section{LA RÉHABILITATION AUDITIVદ عN CHIFFRES}

Prothèse ossiculaire

Le prix de la prothèse ossiculaire est inclus dans celui de l'acte chirurgical

Aide auditive conventionnelle

- $1600 €$

Implant d'oreille moyenne

- Implant: $6200 €$

- Processeur externe: $2800 €$ à la charge du patient

- Suivi orthophonique selon la sévérité de l'atteinte

Implant cochléaire

- Implant: $23000 €$

- Bilan pré-opératoire: audiométrie, bilans radiologique, orthophonique et psychologique

- Suivi orthophonique des adultes:

- règlages: 10 environ, cotation K25 (idem qu'un audiogramme: $\sim 50 €$ )

- bilans: 3 la $1^{\text {re }}$ année, l/an ensuite, cotation AM015 $(\mathrm{AMO}=2,35 €)$

- rééducation orthophonique: 40 séances, cotation AM015

- Suivi orthophonique des enfants: prise en charge médicale, éducative, sociale..

Implant du tronc cérébral

- Implant: $23000 €$

- Bilan pré-opératoire: audiométrie, bilans radiologique, orthophonique et psychologique

- Suivi orthophonique (adultes):

- règlages: 10 environ, cotation K25 (idem qu'un audiogramme: $50 €)$

- bilans: 3 la $1^{\text {re }}$ année, $1 /$ an ensuite, cotation AM015 $(\mathrm{AMO}=2,35 €)$

- rééducation orthophonique: 80 séances, cotation AM015

Prothèse à ancrage osseux

- Pilier externe: $695 €$

-Prothèse: modèle classique: $2300 €$

- modèle Cordell : $2673 €$

- modèle compact: $2759 €$

Chiffres donnés à titre indicatif

\section{TIRÉS À PART}

D. Bouccara 